\title{
TRANSIENT BIOBATTERIES: MICROFLUIDIC CONTROL FOR PROGRAMMABLE DISSOLUTION
}

Maedeh Mohammadifar and Seokheun Choi*,

Bioelectronics \& Microsystems Laboratory, Department of Electrical \& Computer Engineering,

Center for Research in Advanced Sensing Technologies \& Environmental Sustainability (CREATES),

State University of New York at Binghamton, New York, USA

\begin{abstract}
We report a simple approach to develop transient microbial fuel cells with the capability of dissolving in water after stable power generation within a programmed period. This novel watersoluble biobattery makes use of the integration of a dissolvable paper-based substrate, a simple pencil-drawn graphite anode, and a Prussian-blue (PB) cathode. The device features (i) a low cost transient paper-based platform, (ii) easily accessible electrode materials and simple fabrication steps and (iii) a time-controlled operation by using the number of serpentine microfluidic channels. The biobatteries reached to a maximum power of $0.5 \mu \mathrm{W}$ and a current $15.6 \mu \mathrm{A}$ and achieved full dissolution in less than 60 minutes.
\end{abstract}

\section{INTRODUCTION}

Transient electronics is an emerging technology with the interesting characteristics to physically disappear on demand [1]. Future research efforts in this realm will continue to revolutionize the fields of temporary biomedical implants, environmental friendly electronics, data-secure memory devices, and disposable consumer electronics [2-4]. However, there are significant challenges in developing transient electronics at a system level that contains an integrated, transient power source [5-9]. The transient power source is essential to creating an all transient system that can work independently and self-sustainably $[5,6]$. Despite the vast promise and demand, however, research efforts on transient power sources or energy storage devices were quite limited. Several transient power sources such as lithium-ion batteries and nanogenerators were proposed to degrade into the surrounding environment by the external triggers such as light, temperature, $\mathrm{pH}$, or special liquid $[8,9]$. However, they do not sustain stable performance in a controllable manner and still contain a large amount of non-renewable and non-biodegradable heavy metals and polymers [10-14].

In this work, we report a bacteria-powered biobattery on a dissolvable paper that can be decomposed in a controlled manner after stable operation (Figure 1). Single sheet transient microbial fuel cells (MFCs) were directly created from a 2-D sheet of watersoluble paper consisting of four functional layers; (1) an anode, (2) a reservoir, (3) a proton exchange membrane (PEM), and (4) a cathode (Figure 2) $[15,16]$. The hydrophilic microfluidic channels for microbial energy production were defined with hydrophobic wax boundaries which efficiently delayed the fast destruction of the substrate in water. A pencil-drawn graphite anode and a Prussian blue (PB) cathode were constructed on water-soluble paper [17], and electricity-producing bacterial cells were preinoculated and freeze-dried in the patterned channels. When the bottom of the device was dipped in water, the liquid was transported vertically via capillary force, rehydrating the freezedried cells and activating their respiration by transferring electrons to the anode (Figure 1) $[18,19]$. Simultaneously, transiency was triggered while the water dissolved the paper substrate from the bottom. With the number of serpentine microfluidic channels, the

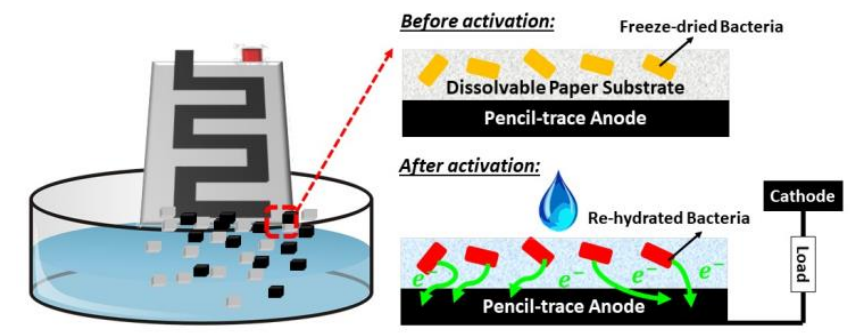

Figure 1: Schematic diagrams of our transient biobattery and its operating principle.
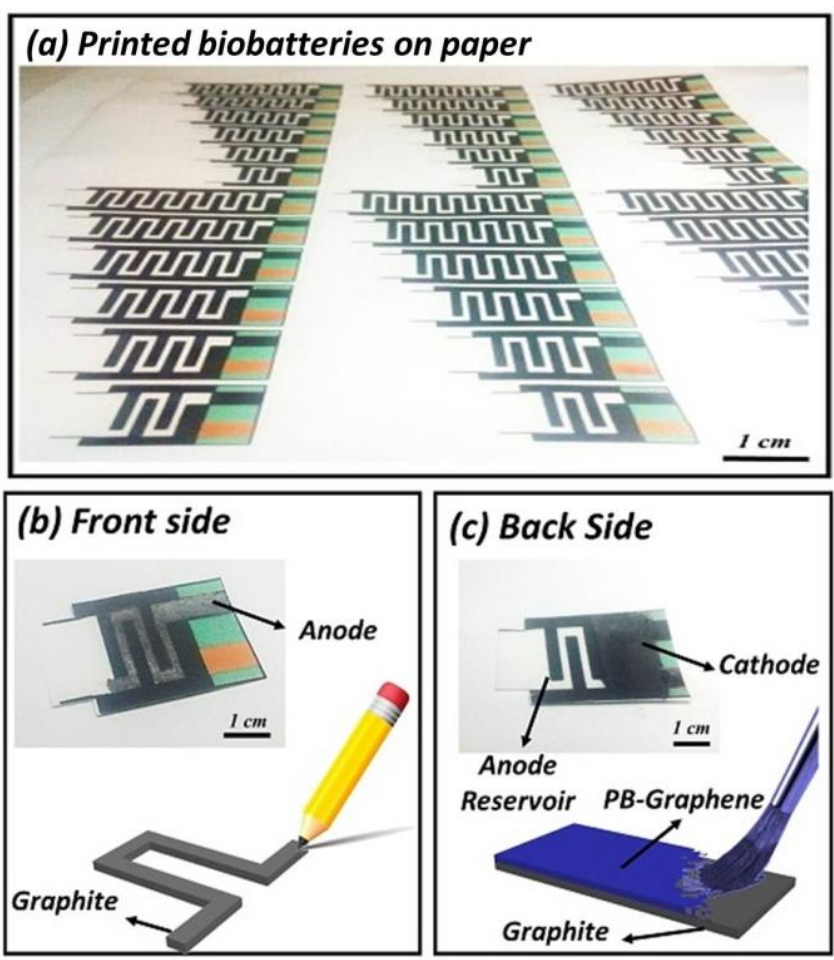

Figure 2: (a) Photo image of the devices with different number of serpentine microfluidic channels $(n=1 \sim 6)$, (b) top and (c) bottom view of the device.

transiency was controlled and programmed. This fabrication and material breakthrough allowed the creation of a novel on-demand transient microbial fuel cell (MFC) platform made only of simple and low-cost materials. A maximum power of $0.5 \mu \mathrm{W}$ and current $15.6 \mu \mathrm{A}$ were achieved.

\section{EXPERIMENTAL SET-UP}

Materials

Poly(3,4-ethylened ioxythiophene):polystyrene sulfonate (PEDOT:PSS), dimethyl sulfoxide (DMSO) and Nafion solution 
were purchased from Sigma Aldrich. Prussian Blue (PB) was obtained from Spectrum Chemical. Graphene was purchased from Angstron Materials, and dissolvable papers were obtained from Uline.

\section{Device fabrication}

Transient MFC devices were fabricated by using a wax printing technique. The hydrophobic wax patterns were designed using AutoCAD software and printed out onto the dissolvable paper using Xerox Phaser printer (ColorQube 8570). The printed paper was then placed in an oven set at $100^{\circ} \mathrm{C}$ for $50 \mathrm{~s}$, and the hydrophobic wax melted into the paper substrate, defining device boundaries and fluidic channels, forming PEMs, and mechanically strengthening the paper to delay rapid dissolving of the paper in water (Figure 2a).

\section{Anodes on paper}

To prepare pencil-graphite anode electrodes, an $\mathrm{HB}$ grade pencil was purchased from American Tombow Inc [20]. The electrodes were prepared by simple drawing on the pre-defined anodic microfluidic channels (Figure 2b). The pencil trace generated the coverage of graphitic layer on top of the reservoir/fluidic channel. The average surface resistance of $310 \Omega$ was obtained throughout the anodic channel.

\section{Cathodes on paper}

The Prussian-Blue (PB)-based cathode was constructed by providing a conductive pencil trace layer on top of the wax-based proton exchange membrane (PEM). The drawn graphite provided the high conductivity of the cathode. The cathode material for each $1 \mathrm{~cm}^{2}$ area was prepared with $8 \mathrm{mg}$ of $\mathrm{PB}$ and $3 \mathrm{mg}$ of graphene in a conductive binder solution followed by ultrasonication for $10 \mathrm{~min}$. The conductive binder solution was prepared with (i) $30 \mu \mathrm{L}$ of PEDOT:PSS solution, (ii) $5 \mu \mathrm{L}$ of $5 \mathrm{wt} \%$ Nafion, (iii) $100 \mu \mathrm{L}$ of isopropanol. The mixture was brush coated on the pre-defined pencil-traced cathodic side of the paper (Figure 2c).

\section{Inoculum}

Shewanella oneidensis MR-1 were grown from $-80^{\circ} \mathrm{C}$ glycerol stock cultures by inoculating $20 \mathrm{~mL}$ of L-broth medium with gentle shaking in air for $24 \mathrm{~h}$ at $35^{\circ} \mathrm{C}$. The L-broth media consisted of $10.0 \mathrm{~g}$ tryptone, $5.0 \mathrm{~g}$ yeast extract and $5.0 \mathrm{~g} \mathrm{NaCl}$ per liter. Both cultures were then centrifuged at $5,000 \mathrm{rpm}$ for $5 \mathrm{~min}$ to remove the supernatant. The bacterial cells were re-suspended in a new medium and used as an anolyte for the device.

\section{Freeze-drying (lyophilization) procedure}

After the MFC devices were inoculated with bacterial cells, they were placed in a low freeze drier (FreeZone Plus 2.5 Liter Cascade Benchtop Freeze Dry System, Labconco, MO, USA), the drying operation was performed at a pressure of $0.06 \mathrm{~atm}$ for $12 \mathrm{~h}$ with freezing and sublimation processes. During the freezing, the chamber temperature dropped to $-50^{\circ} \mathrm{C}$ and then progressively increased back to room temperature. The device was not affected by the lyophilization processes.

\section{Measurement setup}

We measured the potentials between the anodes and the cathodes with a data acquisition system (National instrument, USB-6212), and recorded the readings every $1 \mathrm{~min}$ via a customized LabView interface. An external resistor was connected
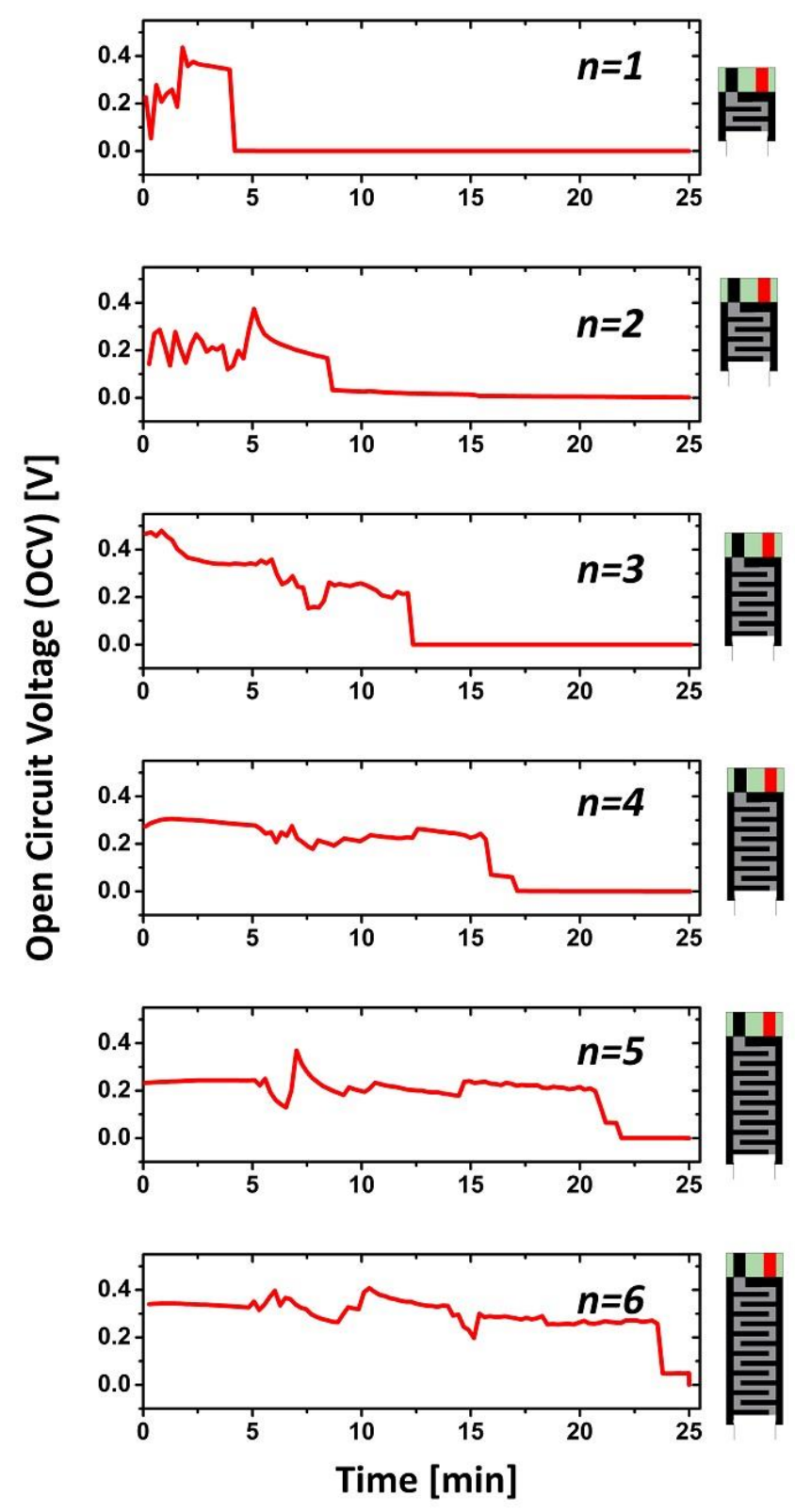

Figure 3: Operation period of the devices with different number of channels.

between the anode and the cathode to close the circuit. The current through this resistor was calculated using Ohm's law.

\section{RESULT AND DISCUSSION}

To effectively control and purposely prolong the device operation time, we defined the different lengths of microfluidic channels. The increase in the length of channels with the waxprinted boundaries as well as the pencil-drawn graphite channels prevented the biobattery from the instant dissolving of battery in the water. During operation, the devices were partially placed in a water container. The freeze-dried bacteria cells were activated through rehydration as the water flowed up the channel while the output voltage and current were monitored. 


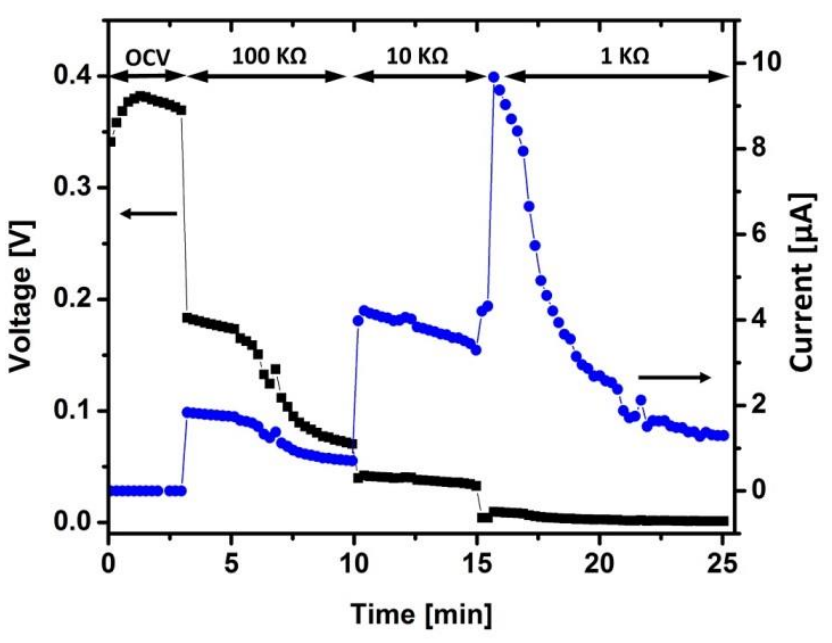

Figure 4: Voltage and current outputs of the transient biobattery $(n=6)$

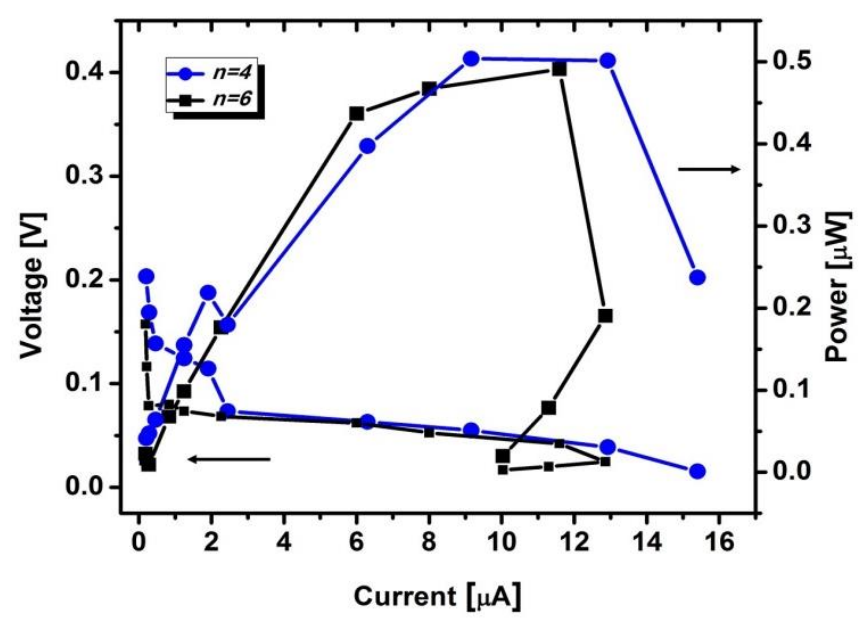

Figure 5: Polarization curveloutput power for the transient biobatteries ( $n=4 \& n=6)$ as a function of current.

\section{Microfluidic channel numbers (n)}

In this work, we tested six different designs with the different number of serpentine microfluidic channel curves $(n=1 \sim 6)$. The open circuit voltage (OCV) for each device was continuously measured during the dissolving process. As shown in Figure 3, the increase in the channel length resulted in the prolonged device operation. The $\mathrm{n}=1$ device operated only for $4 \mathrm{~min}$. while the voltage generation of the $n=6$ one was sustained for more than $22 \mathrm{~min}$. We further tested the $\mathrm{n}=6$ device with three different resistors $(100 \mathrm{~K} \Omega, 10 \mathrm{~K} \Omega$ and $1 \mathrm{~K} \Omega$ ) to measure the current outputs (Figure 4). For $25 \mathrm{~min}$, the device successfully operated, generating current outputs in response to the resistors. Figure 5 shows the polarization curve and the power output of the devices with $n=4$ and $n=6$ channels. A maximum power of $0.5 \mu \mathrm{W}$ and current $15.6 \mu$ A were produced from the biobattery $(n=6)$.

\section{Dissolvability}

The dissolvability of the biobattery and its components (i.e. the substrate, printed wax boundaries and membrane, pencil-
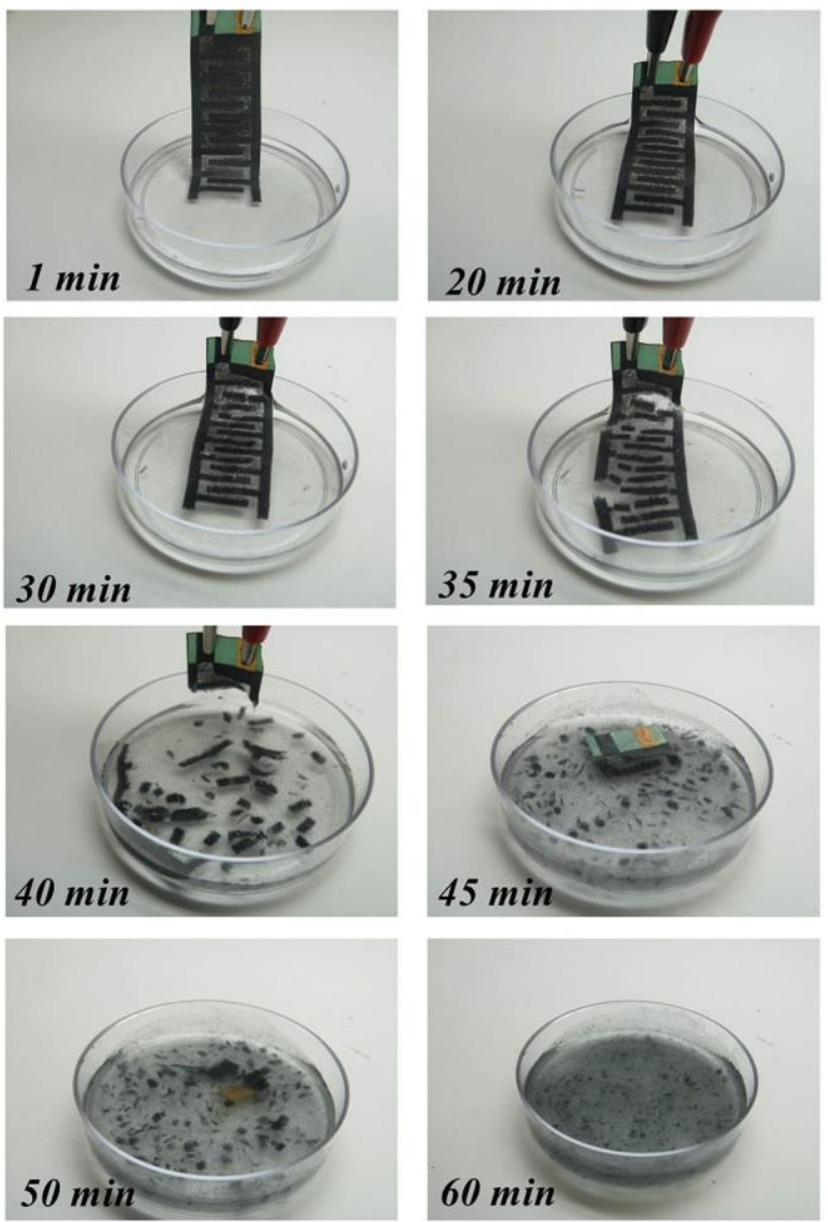

Figure 6: Transient test of the device $(n=6)$ in water. After 60 min, the device completely dissolved in water.

drawn electrodes and Prussian-Blue cathode) were evaluated in water. The substrate itself could completely dissolve within the less than 2 min while the wax-printed boundaries and the pencildrawn traces required more time for the dissolution. Figure 6 demonstrates the dissolving courses of the entire battery with $n=6$ in water at the room temperature. For the complete dissolution of the device, it took about $60 \mathrm{~min}$.

\section{CONCLUSION}

This work provided a novel technique to develop a transient biobattery by using a microfluidic control for programmable dissolution. The transient paper-based MFC used the dissolvable paper as a substrate, the pencil-drawn graphite anode and the Prussian-blue (PB) cathode. The different lengths of the microfluidic channels controlled the operation time of the battery. The freeze-dried bacteria cells pre-loaded on paper can be very useful for on-demand battery operation in resource-limited environments. This work will provide an innovative strategy to develop a transient battery with an easy-to-use and inexpensive alternative fabrication method. 


\section{ACKNOWLEDGMENT}

This work is supported by NSF (ECCS \#1503462 \& ECCS \#1703394) and the SUNY Binghamton Research Foundation (SETAE).

\section{REFERENCES}

[1] K.K. Fu, Z. Wang, J. Dai, M. Carter, L. Hu, "Transient Electronics: Materials and Devices," Chem. Mater., 28, 3527, 2016.

[2] H. Cheng, V. Vepachedu, "Recent development of transient electronics," Theoretical and Applied Mechanics Letters, 6, $21,2016$.

[3] L. Yin, H. Cheng, S. Mao, R. Haasch, Y. Liu, X. Xie, S. Hwang, H. Jain, S. Kang, Y. Su, R. Li, Y. Huang, J.A. Rogers, "Dissolvable metals for transient electronics," Advanced Functional Materials, 24, 645, 2014.

[4] R. Li, H. Cheng, Y. Su, S. Hwang, L. Yin, H. Tao, M.A. Brenckle, D. Kim, F.G. Omenetto, J.A. Rogers," An analytical model of reactive diffusion for transient electronics," Advanced Functional Materials, 23, 3106, 2013.

[5] Y. Chen, R. Jameshidi, K. White, S. Cinar, E. Gallegos, N. Hashemi, R. Montazami, "Physical-chemical hybrid transiency: a fully transient Li-ion battery based on insoluble active materials," Journal of Polymer Science, Part B: Polymer Physics, 54, 2021, 2016.

[6] Y. Kim, S. Chun, J. Whitacre, C.J. Bettinger, "Self-deployable current sources fabricated from edible materials," Journal of Materials Chemistry B, 1, 3781, 2013.

[7] V. Edupuganti, R. Solanki, "Fabrication, characterization, and modeling of a biodegradable battery for transient electronics," Journal of Power Sources, 336, 447, 2016.

[8] K. Fu, et al., "All-component transient lithium-ion batteries," Advanced Energy Materials, 6, 1502496, 2016.

[9] Q. Zheng, Y. Zou, Y. Zhang, Z. Liu, B. Shi, X. Wang, Y. Jin, H. Ouyang, Z. Li, Z.L. Wang, "Biodegradable triboelectric nanogenerator as a life-time designed implantable power source," Science Advances, 2, e1501478, 2016.

[10] J. P. Esquivel, P. Alday, O. A. Ibrahim, B. Fernández, E. Kjeang, N. Sabaté, "A metal-free and biotically degradable battery for portable single-use applications," Advanced Energy Materials, 7, 1700275, 2017.

[11] M. Irimia-Vladu, "Green electronics: biodegradable and biocompatible materials and devices for sustainable future," 43, 588, 2014.

[12] G.A. Salvatore, J. Sulzle, F.D. Valle, G. Cantarella, F. Robotti, P. Jokic, S. Knobelspies, A. Daus, L. Buthe, L. Petti, N. Kirchgessner, R. Hopf, M. Magno, G. Troster, "Biodegradable and highly deformable temperature sensors for the internet of things,"Advanced Functional Materials, 27, 1702390, 2017.

[13] M. Irimia-Vladu, E.D. Glowacki, G. Voss, S. Bauer, N.S. Sariciftci, "Green and biodegradable electronics," Materials Today, 15, 340, 2012.

[14] X. Gao, L. Huang, B. Wang, D. Xu, J. Zhong, Z. Hu, L. Zhang, J. Zhou, "Natural materials assembled, biodegradable, and transparent paper-based electret nanogenerator," ACS Applied Materials \& Interfaces, 8, 35587, 2016.

[15] M. Mohammadifar, J. Zhang, I. Yazgan, O. Sadik, S. Choi, "Power-on-paper: Origami-inspired Fabrication of 3-D Microbial Fuel Cells," Renewable Energy, 118, 695, 2018.

[16] Y. Gao, S. Choi, "Stepping Towards Self-powered Papertronics: Integrating Biobatteries into a Single Sheet of
Paper," Advanced Materials Technologies, 2, 1600194, 2017.

[17] S.A.M. Shaegh, N. Nguyen, S.M.M. Ehteshami, and S.H. Chan, "A membraneless hydrogen peroxide fuel cell using Prussian Blue as cathode material," Energy \& Environmental Science, 5, 8225, 2012.

[18] M. Mohammadifar, S. Choi, "A Papertronic, On-Demand and Disposable Biobattery: Saliva-Activated Electricity Generation from Lyophilized Exoelectrogens Pre-inoculated on Paper," Advanced Materials Technologies, 2, 1700127, 2017.

[19] M. Mohammadifar, S. Choi, "On-demand micro-power generation from an origami-inspired paper biobattery stack," Batteries, 4, 14, 2018

[20] C. Lin, Z. Zhao, J. Kim, J. Huang, "Pencil drawn strain gauges and chemiresistors on paper," Scientific Reports, 4, 3812,2014

\section{CONTACT}

*Seokheun Choi, Assistant Professor, SUNY-Binghamton, Email:sechoi@binghamton.edu Website: http://ws.binghamton.edu/choi 